\title{
How the market responds to dynamically inconsistent preferences
}

\author{
by Ben McQuillin and Robert Sugden* \\ * School of Economics, University of East Anglia, \\ Norwich NR4 7TJ, UK
}

\begin{abstract}
This paper responds to the 'soft paternalist' argument that the findings of behavioural economics make traditional objections to paternalism incoherent. We show that there is a normatively significant sense in which, even if individuals lack coherent preferences, competitive markets are efficient in providing them with opportunities to get what they want. Extending earlier analysis by Sugden, we model a multi-period 'storage economy' and explore the implications of dynamically inconsistent preferences. We show that, despite apparent conflicts of judgement between an individual's 'selves', competitive markets provide maximal opportunity, and that they do so by facilitating voluntary exchanges between selves.
\end{abstract}

JEL classification codes

\section{Keywords}




\section{Introduction}

A common idea can be discerned in many contributions to the literature on reconciling normative and behavioural economics: that incoherence in individuals' preferences justifies new kinds of intervention in markets. The background thought is that if preferences are incoherent, the preference-satisfaction criterion of traditional welfare economics cannot be applied. Thus (it is thought) the presumption against paternalism imparted by that criterion may no longer be justified. This response to behavioural economics is coming to be known as soft paternalism. In this paper, we develop a line of analysis which poses a challenge to soft paternalism by showing that there is a normatively significant sense in which, even if individuals lack coherent preferences, competitive markets are efficient in providing them with opportunities to get what they want.

In one of the best-known statements of the soft paternalist position, Sunstein and Thaler (2003) argue for 'libertarian paternalism' (see also Thaler and Sunstein, 2008). They claim that if individuals lack well-formed preferences, the principle of respecting preferences becomes 'incoherent' and paternalism becomes 'inevitable' (2003, p. 1182). However, they propose that paternalistic interventions should not constrain individual freedom of choice, thus guaranteeing that coherent preferences are respected. In their parallel proposal of 'asymmetric paternalism' Camerer et al (2003) claim that behavioural economics justifies new kinds of market regulation to increase the welfare of 'boundedly rational' individuals. They seek to minimise the costs that this regulation imposes on individuals who are fully rational.

Bernheim and Rangel's (2009) proposals for 'behavioural welfare economics' are based on similar principles. They represent a problem of individual choice (a 'generalised choice situation' or GCS) as a pair $(X, d)$ where $X$ is an opportunity set of 'objects' and $d$ is an 'ancillary condition' (such as the framing of the choice problem). The individual's behaviour is represented by a choice correspondence whose domain is the set of possible GCSs. Bernheim and Rangel use revealed preferences to define a relation of 'unambiguous choice' to be used as a welfare criterion. (Object $x$ is 'unambiguously chosen over' object $y$ if there is no GCS in which $x$ is feasible but $y$ is in the chosen set.) This relation respects revealed preferences that are constant across ancillary conditions but is silent otherwise. 'Welfare optima' (that is, objects which cannot be unambiguously improved on) always exist, but this provides an undiscerning criterion if choices vary greatly across ancillary conditions. Bernheim and Rangel suggest that their criterion can be refined by deleting 
normatively 'suspect' GCSs from the set from which welfare rankings are defined. As they point out, there are similarities between their approach and ideas in the literature of multiple selves. If each ancillary condition is interpreted as activating a different self, and if each self acts on a consistent preference ordering, welfare optimality is equivalent to Pareto optimality among the selves.

In this paper, we develop a very different reconciliation of normative and behavioural economics, building on earlier work by Sugden. Sugden (2004) defines an 'opportunity criterion' which is intended to represent the normative intuition traditionally expressed in appeals to 'consumer sovereignty'. It can be interpreted as the condition that individuals have access to all opportunities for feasible and mutually beneficial transactions, 'benefit' being understood in terms of voluntary choice. In arguing for this criterion, Sugden uses an analysis of time-inconsistent choice that is very different from that of conventional multipleselves models. The essential idea (expanded on in Sugden, 2007) is that a 'responsible' individual identifies with all of her choices, even if those choices cannot be rationalised by a single system of preferences. Using a simple model of an exchange economy, Sugden (2004) shows that the opportunity criterion is satisfied if the 'law of one price' holds (that is, if for each non-money good there is a single price at which it can be freely bought and sold by every individual) and if all markets clear. This result does not depend on any assumptions about the rationality of individuals or the coherence of their preferences. ${ }^{1}$

Sugden also shows that the law of one price and market clearing are equilibrium conditions for a market in which trades between individuals are mediated by a distinct class of rational profit-seeking arbitrageurs, and he proves the existence of such an equilibrium. These results hold under very mild assumptions about individual behaviour. The only substantive assumption about the rationality of individuals is that they are 'price-sensitive': at any given moment, they buy only at the lowest of the prices currently posted by arbitrageurs and sell only at the highest. The implication is that competitive markets are able to satisfy the opportunity criterion even if preferences are incoherent.

One of the simplifying features of Sugden's model is that exchange takes place in an extended 'trading period' which precedes any consumption. Individual consumers may reveal preference inconsistency by making trades at earlier moments in the trading period

\footnotetext{
${ }^{1}$ The idea that competitive equilibrium can be characterised in terms of mutually beneficial transactions is also developed by Hammond (2003), but under the assumption of coherent preferences.
} 
which they reverse at later moments, but (because of the effects of arbitrage) such reversals can be made without loss. Actual consumption is effectively determined by decisions made at the final moment. Thus, each individual's final consumption decision can be interpreted as a chosen point on a budget constraint, as if that individual had been acting on preferences that satisfied the standard neoclassical assumptions.

Because of this feature, Sugden's analysis might be thought not to address some of the most important normative issues raised by the findings of behavioural economics. A recurring idea in recent behavioural literature (and exemplified by Bernheim and Rangel's analysis) is that when an individual lacks coherent preferences, her actions can usefully be modelled in terms of multiple selves. Such models typically induce individual behaviour that is dynamically inconsistent, and the supposedly undesirable properties of such inconsistency can be interpreted as justifying paternalistic interventions. However, if one describes the workings of Sugden's model in the language of multiple selves, the pattern of consumption is as if the preferences of the self acting at the last moment had been respected and those of all other selves had been ignored. Thus, inconsistencies between the preferences of different selves cannot induce anomalous effects on consumption.

A further problem is that Sugden's opportunity criterion refers only to opportunities to consume and not (or not directly) to opportunities to trade. Within his model, however, the space in which dynamic inconsistencies can be revealed is the space of trading opportunities. (They are revealed when an individual makes some exchange at one moment of the trading period and then reverses that exchange at a later moment.) Given that the law of one price holds, a market in which trade takes place over an extended period gives each individual unlimited opportunities to make dynamically inconsistent trades. It is natural to ask whether such opportunities are normatively significant; but to address this question one needs a concept of opportunity that refers not only to consumption, but also to trade.

Our object in this paper is to extend Sugden's approach so as to allow an analysis of dynamic inconsistency. We do this by generalising Sugden's model economy and by reformulating the opportunity criterion.

In specifying a model economy, our strategy is to retain as much as possible of the simplicity of the one-period exchange-economy model that has traditionally served as the starting point for normative analyses of the market system. However, we need a model in which dynamic inconsistency can have effects on consumption. This requires that 
individuals engage in economic activity in at least two periods, and that actions taken in one period can have irreversible effects on the economy-wide feasibility constraints that are binding in later periods. For these reasons, we develop a model of a 'storage economy'. A storage economy exists over a succession of periods. As in an exchange economy, there is no production. For each good, the total stock (and, since we do not allow 'free disposal', the total consumption) of each good is fixed. The stock of a good may be capable of being consumed in more than one period. This provides a relatively simple modelling framework in which consumption decisions made in earlier periods can impact on the feasibility constraints of later periods. The exchange economy analysed by Sugden can be interpreted as a special case of a storage economy in which goods can be traded in all periods but consumed only in the final period.

The opportunities available in our model economy are defined as constraints within which, over successive periods, individuals make decisions about their holdings and consumption of goods. Different economic institutions or 'regimes' are represented by different sets of constraints, a competitive market (with a given array of prices) being one such regime. In the interests of brevity, we treat the law of one price and market clearing as defining properties of the market regime. We show that, in all storage economies, the market regime satisfies our opportunity criterion.

To help the reader to gain intuition about our general results, we also describe and analyse a particularly simple special case of the model, the 'wine economy'. In this economy, individuals' preferences are well-defined but dynamically inconsistent. In the language of multiple-selves models, there is for each individual an inconsistency between the preferences of an earlier and a later self. Because of this inconsistency, consumption patterns in a market regime are different from those in a regime of autarky, or in a regime in which individuals precommit their future consumption. The two selves have opposing evaluations of these consumption patterns. But, while is it is an implication of our general result that the market regime satisfies the opportunity criterion, neither of the other regimes has this property. The simplicity of the wine economy helps one to understand the sense in which opportunities to make dynamically inconsistent trades can be normatively significant. We argue that the market is mediating trades between different selves of the same individual. In this sense, the market provides opportunities for mutually beneficial (or voluntarily chosen) transactions. 


\section{Opportunity in a storage economy}

Our model of a storage economy is made up of individuals $i=1, \ldots, N$, infinitely divisible goods $g=1, \ldots, G$, and periods $t=1, \ldots, T$. Each individual is economically active in every period. For each good $g$, there is a non-empty set of periods $Z_{g} \subseteq\{1, \ldots, T\}$ in which the good is consumable $;^{2}$ the last period in which it is consumable is denoted $z_{g}$, after which it has expired. (As an aid to intuition, imagine an economy which lasts from January [period 1] to December [period 12]. The goods are fruits which grow spontaneously and can be harvested with no expenditure of effort. Good $g$ might be a fruit which appears on trees in a fixed quantity in July and can be picked at any time up to September, after which it rots. Then $Z_{g}=\{7,8,9\}$ and $z_{g}=9$.) At the start of period 1, each individual $i$ has a non-negative endowment $e_{i, g}$ of claims on each good $g$. A claim on a unit of good $g$ can be roughly interpreted as an entitlement to consume one unit of that good in some period in $Z_{g}$, the holder of the claim being free to choose the period of consumption. ${ }^{3}$

In the economy of our model, there is no option of free disposal. ${ }^{4}$ Instead, we require that, for each good $g$, all claims on $g$ are converted into consumption on or before the expiry period $z_{g}$. 'Consumption' need not be interpreted as something that individuals value positively. For our purposes, consumption is an act by an individual which retires or extinguishes units of a good; this act might be valued positively or negatively. (In the fruit tree economy, 'consumption' might involve the enjoyment of eating a desirable fruit or the inconvenience of composting an undesirable one.) Thus, a claim on a good carries both an entitlement and an obligation to consume. However, we will interpret good 1 (money) as a good whose consumption is always valued positively. ${ }^{5}$ We stipulate that $Z_{1}=\{1, \ldots, T\}$, thus ensuring that good 1 can serve as a standard of value and a means of exchange in all periods.

\footnotetext{
${ }^{2}$ We use $\subseteq$ to denote 'is a weak subset of' and reserve $\subset$ for 'is a strict subset of'.

${ }^{3}$ This need not be interpreted as implying that storage costs are zero. Formally, our assumption is that (up to the period of expiry) one 'unit' of good $g$ in one period can be transformed into one 'unit' of the same good in the next period. From one period to the next, however, the physical dimensions of a unit might shrink (corresponding to storage costs) or grow (as might be the case for unpicked fruit).

${ }^{4}$ Sugden (2004) assumes free disposal. In Appendix 2, we explain why that assumption is necessary given Sugden's formulation of the opportunity criterion and why it is not necessary given ours.

${ }^{5}$ This is a matter of interpretation and not a formal property of our model, because the concept of preference does not appear in our analysis.
} 
Our aim is to assess alternative systems of economic organisation (or 'regimes'), as applied to our model economy. Our strategy is to define a regime in terms of the opportunities it confers on individuals. This strategy requires us to be able to represent very different regimes in a common theoretical framework. The framework we use, and which we now describe, imposes few substantive restrictions on the properties of regimes; essentially, it is a system of accounting conventions.

Consider any individual $i$ in any period $t$. At the start of the period, $i$ has an inheritance $q_{i, g}^{t}$ of claims on each good $g$. Inheritances in period 1 are equal to endowments, and hence are non-negative. Depending on the specification of the regime, $i$ may have opportunities to (or be compelled to) acquire additional claims on non-expired goods and/or disburse existing claims. Her net acquisition (that is, acquisition minus disbursement) of $\operatorname{good} g$ in period $t$ is denoted $\Delta_{i, g}^{t}$. She may also have opportunities to (or be compelled to) convert claims on non-expired goods into consumption. Her consumption of good $g$ in period $t$ is denoted $\Lambda_{i, g}^{t}$, which is required to be non-negative. If good $g$ expires in period $t$ (i.e. if $t=z_{g}$ ), consumption must be equal to inheritance plus net acquisition; otherwise, consumption may be less than this, but cannot be more. ${ }^{6}$ Claims on $g$ that have not been converted into consumption in period $t$ become $i$ 's inheritance in period $t+1$.

The definitions and assumptions stated so far can be represented as:

$$
\begin{array}{ll}
\text { for all } i, g: & q_{i, g}^{1}=e_{i, g} \geq 0 \\
\text { for all } i, g, t \text { where } t<z_{g}: & \Lambda_{i, g}^{t}-\Delta_{i, g}^{t} \leq q_{i, g}^{t} \\
\text { for all } i, g, t \text { where } t=z_{g}: & \Lambda^{t}{ }_{i, g}-\Delta_{i, g}^{t}=q^{t}{ }_{i, g} \\
\text { for all } i, g, t \text { where } t \in Z_{g}: & \Lambda_{i, g}^{t} \geq 0 \\
\text { for all } i, g, t \text { where } t \notin Z_{g}: & \Lambda_{i, g}^{t}=0 \\
\text { for all } i, g, t \text { where } t>z_{g}: & \Delta_{i, g}^{t}=0 \\
\text { for all } i, g, t \text { where } t<T: & q^{t+1}{ }_{i, g}=q^{t}{ }_{i, g}+\Delta_{i, g}^{t}-\Lambda_{i, g}^{t}
\end{array}
$$

It follows from (1) to (5) that:

$$
\begin{array}{ll}
\text { for all } i, g, t \text { where } t \leq z_{g}: & q_{i, g}^{t} \geq 0 \\
\text { for all } i, g, t \text { where } t>z_{g}: & q_{i, g}^{t}=0
\end{array}
$$

\footnotetext{
${ }^{6}$ This restriction prevents individuals from selling short (or, equivalently, in engaging in unsecured borrowing). Our modelling strategy separates the role of consumer (represented by our 'individuals') from that of arbitrageur. When modelling a market regime, we implicitly assume that exchanges are mediated by arbitrageurs, who are allowed to sell short.
} 
The net acquisitions and consumption made by any individual $i$ in any period $t$ can be described by a vector $b_{i}^{t} \equiv\left(\Delta^{t}{ }_{i, 1}, \ldots, \Delta_{i, G}^{t} ; \Lambda_{i, 1}^{t}, \ldots, \Lambda_{i, G}^{t}\right)$; this is a $t$-period behaviour. We now define a dominance relation among such behaviours:

Dominance. Consider any individual $i$ and any period $t$. Let $b_{i}^{t}=\left(\Delta_{i, 1}^{t}, \ldots, \Delta_{i, G}^{t}\right.$; $\left.\Lambda_{i, 1}^{t}, \ldots, \Lambda_{i, G}^{t}\right)$ and $\underline{b}_{i}^{t}=\left(\underline{\Delta}_{i, 1}^{t}, \ldots, \underline{\Delta}_{i, G}^{t} ; \underline{\Lambda}_{i, 1}^{t}, \ldots, \underline{\Lambda}_{i, G}^{t}\right)$ be any $t$-period behaviours for $i$, such that (i) for all $g=2, \ldots, G, \underline{\Delta}_{i, g}^{t}=\Delta_{i, g}^{t}$ and $\underline{\Lambda}_{i, g}^{t}=\Lambda_{i, g}^{t}$, and (ii) $\underline{\Delta}_{i, 1}^{t}-\underline{\Lambda}_{i, 1}^{t}=\Delta_{i, 1}^{t}$ $-\Lambda_{i, 1}^{t}$. Then $\underline{b}_{i}^{t}$ strictly dominates (respectively: weakly dominates) $b_{i}^{t}$ if $\underline{\Lambda}_{i, 1}^{t}>\Lambda_{i, 1}^{t}$ (respectively: $\underline{\Lambda}_{i, 1}^{t} \geq \Lambda_{i, 1}^{t}$ ).

Thus, $\underline{b}_{i}^{t}$ strictly (weakly) dominates $b_{i}^{t}$ if the two behaviours differ only in respect of the net acquisition and consumption of money, if the net change in $i$ 's holding of money during period $t$ is the same in both behaviours, and if $i$ 's consumption of money is strictly (weakly) greater in $\underline{b}_{i}^{t}$. Given our interpretation of money as a good whose consumption is always desirable, a strictly dominating behaviour is unambiguously more desirable than the behaviour it dominates.

We will represent specific regimes in terms of individuals' opportunities for net acquisition and consumption. We define a t-period opportunity set for individual $i$, denoted $O_{i}^{t}$, as a non-empty set of $t$-period behaviours for $i$; the interpretation is that if $i$ faces this set in period $t$, she chooses one and only one of its elements. Since the opportunities open to $i$ in any period will typically depend on her inheritances in that period, we define a t-period opportunity function for individual $i$ as a function, denoted $f_{i}^{t}$, which assigns a $t$-period opportunity set $O_{i}^{t}$ to each vector of $t$-period inheritances $q_{i}^{t} \equiv\left(q_{i, 1}^{t}, \ldots, q^{t}{ }_{i, G}\right)$ that is consistent with (6a) and (6b); we require that each $b_{i}^{t} \in O_{i}^{t}$ is consistent with (2a)-(4), given $q_{i}^{t}$. A regime (typically $R$ ) is defined as an $N \times T$ array of $t$-period opportunity functions, one function $f^{t}{ }_{i}$ for each individual $i$ in each period $t .^{7}$

\footnotetext{
${ }^{7}$ Notice that an individual's decisions in one period can affect the extent of her opportunities in later periods, but that all such effects are transmitted through inheritances. This feature of our framework is less restrictive that it might appear at first sight, since many forms of precommitment can be represented by using suitably-defined goods. For example, suppose one wants to model a threeperiod economy in which, in period 1 , an individual $i$ can choose whether or not to subject herself to the specific constraint that, in period 3, her net acquisition of some good $g$ zero. As a means of recording $i$ 's decision, we can define a good $k$ with $Z_{k}=\{1,2,3\}$ and $e_{i, k}=1$, and interpret a non-zero holding of this good by $i$ as signalling a wish to be subject to the specific constraint. (Think of good $k$ as a written statement of that wish, and 'consumption' of this good as destroying the statement.) Now consider a regime in which $\Delta_{i, k}^{t}=0$ for all $t, \Lambda_{i, k}^{2}=0$, and $O_{i}^{3}$ is defined so that $\Delta_{i, g}^{3}=0$ if $q^{3}{ }_{k}>$
} 
Consider any regime $R$. A $t$-period behaviour $b_{i}^{t}$ for individual $i$ is allowable in $R$, given an inheritance $q_{i}^{t}$, if it is consistent with $f_{i}^{t}$, as specified by $R$, and with (2a)-(4). We define a lifetime behaviour for $i$ as a vector $b_{i} \equiv\left(b^{1}, \ldots, b^{T}{ }_{i}\right)$ of $t$-period behaviours. A lifetime behaviour $b_{i}$ is allowable in $R$ if it is consistent with $f^{1}{ }_{i}, \ldots, f^{T}$, as specified by $R$, and with (1)-(5). We define an outcome as a vector $b \equiv\left(b_{1}, \ldots, b_{N}\right)$ of lifetime behaviours, one for each individual. An outcome $b$ is allowable in $R$ if each $b_{i}$ is allowable. The set of allowable outcomes for $R$ will be denoted $O(R)$.

For any regime $R$, an outcome $o$ is non-dominated in $O(R)$ if, for each individual $i$ and each period $t$, the $t$-period behaviour $b_{i}^{t}$ implied by $o$ is not strictly dominated by any $t$ period behaviour that is allowable in $R$, given the inheritance $q_{i}^{t}$ implied by $o$. Intuitively, an outcome is non-dominated if, in each period considered separately, no individual passes up an opportunity to consume more money. ${ }^{8}$

An outcome is feasible if, for each good $g, \Sigma_{i} \Sigma_{t} \Delta_{i, g}^{t}=0$. (Equivalently, total consumption of each good is equal to total endowments.) Notice that allowable outcomes are not necessarily feasible: allowability is defined in relation to constraints which apply to individuals separately, while feasibility is a property of individuals' decisions in conjunction.

We shall assume that, for any given regime, each individual's lifetime behaviour is uniquely determined. Thus, for any regime $R$, a unique outcome $\chi(R) \in O(R)$ is determined by the decisions that individuals make within that regime's constraints; $\chi(R)$ is the realised outcome of regime $R$. In general, however, we shall not assume that individuals' lifetime behaviours are chosen rationally.

We can now define an opportunity-based normative criterion against which any regime can be assessed: ${ }^{9}$

The opportunity criterion. Consider any regime $R$ and realised outcome $\chi(R)$. Let $\Omega(R) \subset O(R)$ be the set of outcomes of $R$ that are allowable, non-dominated and

0 . By choosing not to consume the whole of her endowment of good $k$ in period $1, i$ imposes on herself the constraint $\Delta_{i, g}^{3}=0$.

${ }^{8}$ This definition does not require that individuals' lifetime behaviours maximise the consumption of money, holding other consumption constant. An outcome can be non-dominated even if individuals' lifetime behaviours reveal dynamic inconsistencies, and even if those inconsistencies result in unambiguous lifetime losses of money.

${ }^{9}$ This criterion reformulates and generalises the criterion proposed by Sugden (2004) for a oneperiod economy. The relationship between the two criteria is discussed in more detail in Appendix 2. 
feasible. $R$ satisfies the opportunity criterion if and only if $\chi(R)$ is feasible and, for every regime $R^{\prime},\left[O\left(R^{\prime}\right) \supset O(R)\right] \Rightarrow\left[\Omega\left(R^{\prime}\right) \subset O(R)\right]$.

To understand the normative intuition behind the opportunity criterion, consider a regime $R$ whose realised outcome $\chi(R)$ is feasible. Thus, the opportunities specified by $O(R)$ can be made available to individuals without any breach of feasibility constraints. Then to say that $R$ satisfies the opportunity criterion is to say that it is not possible to relax the constraints that $R$ imposes on individuals (thus expanding the set of allowable outcomes) in such a way that some feasible outcome, not allowable in $R$, becomes both allowable and non-dominated. In other words, if every individual is to have at least the opportunities that she enjoys in $R$, it is not possible to make available any further non-dominated opportunities which, if taken up, would be compatible with feasibility constraints.

We now characterise a particular type of regime for a storage economy, which we will call a single-price regime. For each non-money good $g$ there is a market price $p_{g}$ (which may be positive, zero or negative). It is convenient to represent the idea that money is the medium of exchange by defining $p_{1} \equiv 1$. In each period $t$, each non-expired non-money good $g$ can be exchanged for money at the market price $p_{g}$. Thus, opportunity functions can be defined as follows. For each individual $i$, for each period $t, f^{t}{ }_{i}\left(q_{i}^{t}\right)$ is the set of $t$-period behaviours for $i$ which satisfy (2a)-(4) and the constraint that the market value of net acquisitions is zero:

$$
\sum_{\mathrm{g}} p_{g} \Delta_{i, g}^{t}=0
$$

A single-price regime is market-clearing if its realised outcome is feasible.

In a one-period economy, the law of one price and the absence of excess supply or demand can be interpreted as equilibrium conditions for a market in which individuals are price-sensitive and in which all trades are mediated by profit-seeking arbitrageurs (Sugden, 2004). Given that storage is costless, constancy of prices across periods is a necessary condition for the absence of unexploited opportunities for profitable arbitrage. Thus, without presupposing that individuals act on coherent preferences, we propose to interpret a marketclearing single-price regime as an equilibrium state of a competitive market. In using this interpretation, we are effectively assuming that, in equilibrium, arbitrageurs can predict the aggregate market behaviour of individuals in future periods - even if individuals themselves fail to predict or to allow for future changes in their own preferences. We suggest that this is 
an acceptable idealising assumption when modelling how professional traders respond to systematic and predictable patterns of individual behaviour of the kinds studied in behavioural economics. (For example, consider problems of self-control. One might expect an experienced cafeteria manager to be able to predict actual daily demands for his products, conditional on how they are displayed, even if most customers consistently buy more cakes and less fruit than they intended before they approached the counter.) Notice also that the problems that we are now supposing our arbitrageurs to be able to solve are much less demanding than those facing the planners who are supposed to design the interventions required by soft paternalism. (Arbitrageurs and planners both need to be able to predict behaviour that is unplanned and unanticipated by the individuals concerned; but planners also need to be able to infer individuals' 'true', unrevealed preferences.)

In this paper, we will not try to translate the intuitive ideas expressed in the previous paragraph into formal theorems. Instead, we will work with the theoretical concept of a market-clearing single-price regime. Our main result (proved in Appendix 1) is a theorem about such regimes:

Market Opportunity Result. For every storage economy, every market-clearing single-price regime satisfies the opportunity criterion.

Before discussing this result in general terms, we will explore its implications for a specific model of a simple storage economy.

\section{The wine economy}

The wine economy is a special case of a storage economy, with $T=2, G=2$ and $N>1$. The non-money good, wine, can be consumed in both periods (i.e. $Z_{g}=\{1,2\}$ ). All individuals are identical. Each individual is endowed with one unit of each good (i.e. $e_{i, 1}=1, e_{i, 2}=1$ ). We shall be primarily concerned with three regimes.

In the autarky regime, there is no interaction between individuals, and consequently no mechanism for the acquisition or disbursement of claims. Each individual's only decisions, made in period 1 , determine how much wine and money she consumes in that period and therefore how much of each good is stored to be consumed in period 2. Thus, autarky is represented by the constraints

$$
\text { for all } i, \mathrm{~g}, t: \quad \Delta_{i, g}^{t}=0 .
$$


These constraints, together with (1)-(5), fully determine the regime's opportunity functions.

In the market regime, each period has a market in which claims on wine are traded against claims on money. We treat as part of the specification of the market regime that there is a single market price of wine in terms of money, denoted $p^{\mathrm{M}}$, at which every individual can trade in both periods and at which the market clears. Thus, the market regime is a single-price market-clearing regime, as defined in Section 2. Its opportunity functions are fully specified by (1) - (5) and:

$$
\begin{array}{ll}
\text { for all } i: & \Delta_{i, 1}^{1}+\mathrm{p}^{\mathrm{M}} \Delta_{i, 2}^{1}=0 \\
\text { for all } i: & \Delta^{2}{ }_{i, 1}+\mathrm{p}^{\mathrm{M}} \Delta_{i, 2}^{2}=0 .
\end{array}
$$

The precommitment regime differs from the market regime in only one respect: there is no market in period 2. Thus, each individual's consumption of the two goods in period 2 is fixed by decisions made in period 1 . Using $p^{\mathrm{P}}$ to denote the market-clearing precommitment price of wine in period 1, the opportunity functions of the precommitment regime are fully specified by (1)-(5) and:

$$
\begin{array}{ll}
\text { for all } i: & \Delta_{i, 1}^{1}+p^{\mathrm{P}} \Delta_{i, 2}^{1}=0 \\
\text { for all } i, g: & \Delta_{i, g}^{2}=0 .
\end{array}
$$

We now specify the particular assumptions that we make about preferences and behaviour in the wine economy. In period 1, each individual's preferences over lifetime behaviours are represented by the utility function

$$
u^{1}=\gamma\left[\alpha \ln \Lambda_{i, 1}^{1}+(1-\alpha) \ln \Lambda_{i, 2}^{1}\right]+(1-\gamma)\left[\beta \ln \Lambda_{i, 1}^{2}+(1-\beta) \ln \Lambda_{i, 2}^{2}\right]
$$

with $\alpha, \beta, \gamma \in(0,1)$. In period 2 , her preferences are represented by the utility function

$$
u^{2}=\gamma^{\prime}\left[\alpha^{\prime} \ln \Lambda_{i, 1}^{1}+\left(1-\alpha^{\prime}\right) \ln \Lambda_{i, 2}^{1}\right]+\left(1-\gamma^{\prime}\right)\left[\beta^{\prime} \ln \Lambda_{i, 1}^{2}+\left(1-\beta^{\prime}\right) \ln \Lambda_{i, 2}^{2}\right]
$$

with $\alpha^{\prime}, \beta^{\prime}, \gamma^{\prime} \in(0,1)$. This specification of period 2 preferences includes an evaluation of period 1 consumption. As this consumption has already taken place and appears in a constant term in (14), it has no effect on period 2 decisions. For normative purposes, however, one might want to represent the judgements that the period 2 self makes about lifetime well-being, and this requires backward-looking as well as forward-looking evaluations. 
The individual's preferences are dynamically consistent if and only if $\beta^{\prime}=\beta^{10} \mathrm{We}$ consider a case of dynamic inconsistency, with $\beta^{\prime}<\beta$. This assumption implies that the weight given to period 2 wine consumption, relative to period 2 money consumption, is higher in $u^{2}$ than in $u^{1}$. (Perhaps the wine improves with age in a way that the individual does not foresee. Or perhaps she develops an unforeseen habituation to alcohol. We leave open the question of whether, had the individual foreseen the change, she would have wanted to accommodate it or to frustrate it.) From now on, to simplify the exposition, we set $\alpha=\alpha^{\prime}=3 / 4, \beta=3 / 4, \beta^{\prime}=1 / 4$, and $\gamma=\gamma^{\prime}=1 / 2$.

We further assume that each individual is myopic in the following sense. In period 1, she takes no account of any opportunities for the acquisition or disbursement of claims that may be available in period 2. Since there are no such opportunities in the autarky or precommitment regimes, this assumption has implications only for the market regime. In other respects, individuals' decisions are utility-maximising.

Thus, in the autarky regime, each individual's lifetime behaviour maximises $u^{1}$ subject to (1)-(5) and (8). In the precommitment regime, each individual's lifetime behaviour maximises $u^{1}$ subject to (1)-(5), (11) and (12), with $p^{\mathrm{P}}$ treated as a constant; the value of $p^{\mathrm{P}}$ is determined by the condition that net acquisitions of each good sum to zero. In the market regime, each individual's period 1 decisions maximise $u^{1}$ subject to (1)-(5), (9) and the perceived (but not actually operative) constraint (12), with $p^{\mathrm{M}}$ treated as a constant. These decisions fix the values of the period 2 inheritances $q_{i, 1}^{2}$ and $q_{i, 2}^{2}$. Taking those values as given, her period 2 decisions select an allowable lifetime behaviour to maximise $u^{2}$, subject to (1)-(5) and (10), again with $p^{\mathrm{M}}$ treated as a constant. The value of $p^{\mathrm{M}}$ is determined by the condition that net acquisitions of each good sum to zero over the two periods taken together.

Solving these constrained maximisation problems is straightforward. The resulting prices and lifetime behaviours for each individual $i$ in each regime are shown in Table 1. The autarky and precommitment regimes induce the same lifetime behaviour, in which consumption of each good is divided equally between the two periods. (The market-clearing

\footnotetext{
${ }^{10}$ The functional form of (14) implies that behaviour in period 2 is independent of the values of $\alpha^{\prime}$ and $\gamma^{\prime}$. It is standard to define dynamic consistency in terms of properties of preferences that are revealed in choices.
} 
equilibrium of the precommitment market is one in which no trade takes place.) However, the market regime induces a different outcome.

\section{[Table 1 near here]}

The price of wine is higher in the market regime $\left(p^{\mathrm{M}}=1\right)$ than in the precommitment regime $\left(p^{\mathrm{P}}=1 / 3\right)$. This higher price induces individuals to consume less wine and more money in period 1, and also to plan to consume less wine and more money in period 2. At the level of the whole economy, it is not feasible for these plans to be realised. However, because of short-selling by arbitrageurs, the period 1 behaviour that these plans entail is realisable. At the end of period 1, arbitrageurs hold claims on N/2 units of wine, matched by liabilities of $N / 2$ units of money. In period 2, individuals find that their plans are no longer utility-maximising, and so engage in further trade, giving up claims on money to acquire additional claims on wine. After trade in period 2, arbitrageurs' holdings of both goods are zero, indicating that the market outcome is feasible. Relative to the autarky and precommitment regimes, the market responds to individuals' dynamic inconsistency by inducing them to behave in period 1 as if they were providing for the preferences they will in fact have in period 2. We now consider the normative significance of this property of the market.

A multiple-selves analysis, of the kind proposed by Bernheim and Rangel, cannot rank the two alternative outcomes (unless the choice problem faced by one of the selves is treated as normatively suspect and 'deleted'). As Table 1 shows, $u^{1}$ is higher in the autarky and precommitment regimes than in the market regime, while $u^{2}$ is lower. In other words, the period 1 self 'prefers' the autarky/precommitment outcome, while the period 2 self 'prefers' the market outcome. ${ }^{11}$ However, the market regime satisfies the opportunity criterion while the other two regimes do not.

That the market regime satisfies the opportunity criterion is an immediate corollary of the Market Opportunity Result. To show that the autarky regime does not satisfy that criterion, let $R$ and $R^{\prime}$ be the autarky and market regimes respectively. Clearly, $O\left(R^{\prime}\right) \supset$

\footnotetext{
${ }^{11}$ Each regime generates a multiple-selves Pareto optimum. Considering only feasible outcomes that are symmetrical with respect to individuals, the outcome induced by autarky and precommitment maximises $u^{1}$ while the market outcome maximises $u^{2}$. The former result reflects the fact that the autarky and precommitment regimes allow the period 1 self to impose its preferred outcome on the period 2 self. The latter result depends on the assumptions that $\alpha=\alpha^{\prime}$ and $\gamma=\gamma^{\prime}$, which imply that there are no conflicts of interest between the selves with respect to the decisions that, in the market regime, are taken by the period 1 self.
} 
$O(R)$; and $\chi\left(R^{\prime}\right)$, the realised outcome of the market regime, is feasible and non-dominated. In other words, substituting the market for autarky expands the set of allowable outcomes, adding at least one outcome that is feasible and non-dominated. To show that the precommitment regime $R^{\prime}$ does not satisfy the opportunity criterion, let $R^{\prime \prime}$ be the regime in which individuals are free to trade at the price $p^{\mathrm{P}}$ in both periods. Clearly, $O\left(R^{\prime \prime}\right) \supset O\left(R^{\prime}\right)$. Consider any feasible outcome $o^{\prime \prime} \in O\left(R^{\prime \prime}\right)$ such that, for at least one individual $i, \Delta_{i, 1}^{2} \neq 0$. Many such outcomes exist, all of which are non-dominated. In other words, substituting $R^{\prime \prime}$ for the precommitment regime expands the set of allowable outcomes, adding outcomes that are feasible and non-dominated.

The reader may have noticed that the autarky and precommitment regimes give each individual the opportunity to achieve the same consumption as she achieves in the market regime, namely $\Lambda_{i, 1}^{1}=3 / 4, \Lambda_{i, 2}^{1}=1 / 4, \Lambda_{i, 1}^{2}=1 / 4$ and $\Lambda_{i, 2}^{2}=3 / 4$. Still, the autarky and precommitment regimes deny her the option of giving up $1 / 2$ unit of wine in exchange for $1 / 2$ unit of money in period 1 , and of reversing that exchange in period 2 . This combination of options is available in the market regime, and is taken up. The market, we might say, is catering to preference inconsistency by giving individuals opportunities to make decisions and then reverse them.

Does this kind of opportunity have normative value? According to the opportunity criterion, it does. That criterion reflects a conception of the individual as a continuing person who is the composition of, and who identifies with, all her momentary selves (Sugden, 2004, 2007). This conception is expressed by specifying an individual's opportunities as her set of allowable lifetime behaviours - as the set of things that she can do over time. The 'she' here is to be understood as one continuing person, whether or not her preferences are dynamically consistent. In the market regime, each individual's set of allowable lifetime behaviours includes the opportunity to make certain trades in period 1 and then to reverse those trades in period 2. This is a genuine opportunity for the individual, in the sense that in period 1 she might want to make those trades and that, having made them, she might in period 2 want to reverse them. The opportunity criterion attaches normative value to a person's being free at each moment to do what she then wants.

Some readers may ask whether a person whose preferences are incoherent can meaningfully be said to want anything. For us, it is self-evident that the answer is 'Yes'. In the sense in which we are using the concept, to want something at a given moment is simply 
to will to choose it (subject to its being available). These momentary wants are authored by one continuing person; and this person identifies with them so that the wants are hers.

It might be objected that the precommitment regime allows individuals to do something that is not possible in the market regime, namely to make decisions in period 1 which precommit period 2 consumption. If one takes the conventional multiple-selves perspective, it may seem that the market is denying an opportunity to each individual's period 1 self. Thus (it might be said), any normative comparison between the market and precommitment regimes requires some higher-level adjudication between conflicting welfare judgements made by the period 1 and period 2 selves. But, as we have explained, the opportunity criterion rests on a different understanding of the relationship between a person's momentary selves. Momentary selves are not seen as sources of potentially conflicting judgements that might need to be reconciled at some higher level; to the contrary, the continuing person identifies with the motivations of all her momentary selves, without demanding consistency between them. In the wine economy, relative to the benchmark of autarky, each individual's period 1 self is willing to give up claims on wine in return for claims on money. Each individual's period 2 self is willing to give up claims on money in return for claims on wine. This creates an opportunity for voluntary exchange between an individual's two selves. The market regime facilitates this transaction; the precommitment regime prevents it. Viewing the person as the composition of her momentary selves, one can construe an opportunity for voluntary exchange between her selves as an opportunity for her to do as she chooses.

If opportunity is understood in terms of lifetime behaviours, any constraint on the reversal of previous decisions unambiguously reduces opportunity. Clearly, that conclusion holds for any storage economy. The fact that the precommitment regime of the wine economy fails to satisfy the opportunity criterion is an illustration of that general result.

\section{Conclusion}

Our objective in this paper has been to analyse how competitive markets respond to the kinds of preference inconsistency that have been documented by behavioural economics. We have shown that there is a coherent pattern in the way that markets respond to individuals' preferences, whether or not those preferences themselves are coherent, and that this pattern can be interpreted as the satisfaction of an opportunity-based normative criterion. 
That criterion formalises normative intuitions that have been traditionally been expressed in terms of consumer sovereignty and anti-paternalism. ${ }^{12}$

We must emphasise that, although our analysis has been radically different from that of soft paternalism, we are not asserting that paternalism (soft or otherwise) is always wrong. We present the opportunity criterion as a representation of anti-paternalistic intuitions, but we do not assert that satisfying this criterion trumps other moral imperatives.

For many years, the conventional approach to welfare economics was based on the assumption that individual behaviour revealed consistent preferences and beliefs, and it was generally accepted that competitive markets were Pareto-efficient in satisfying those preferences. Nevertheless, welfare economists often justified particular policy interventions by claiming that individuals' preferences and beliefs, although internally consistent, were imprudent, misguided, ill-informed or mistaken. For example, it was commonly argued that, as a result of 'myopia', individuals' subjective time preference rates were too high; the concept of 'merit goods' was sometimes deployed to justify the supply of supposedly worthy but insufficiently preferred goods. Of course, such arguments had their opponents as well as their proponents. Our point is that consensus on the theoretical question of how competitive markets respond to individuals' preferences did not foreclose normative debate about the merits and demerits of paternalistic policies. However, the proposition that competitive markets are Pareto-efficient provided a point of reference for such debates by making clear that paternalistic interventions in the market are not normatively costless: one of their costs is the disabling of mechanisms that promote the satisfaction of individuals' preferences.

We see our results in a similar light. If the central theoretical claims of this paper were accepted, the arguments for and against specific paternalistic policies would remain matters for debate. But it would be recognised that such policies have a normative cost, even if the individuals whose interests are supposedly being promoted lack the coherent preferences assumed in traditional welfare economics. Contrary to the claims of some soft paternalists, the findings of behavioural economics do not make principled opposition to paternalism incoherent.

\footnotetext{
${ }^{12}$ We do not claim that the opportunity criterion expresses the whole of a defensible account of the normative value of opportunity. One might want to compare the extent of opportunity provided to a given individual by two different opportunity sets, neither of which is a subset of the other. Or one might want to compare the extent of opportunity enjoyed by two different individuals. Sugden (2010) explores the relationship between the opportunity criterion and the principles involved in such comparisons.
} 
In a normatively significant sense, a competitive market in private goods provides maximal opportunities for voluntary transactions - that is, for transactions that individuals choose to make at the time they make them. If an individual's preferences are inconsistent across time, the transactions that the market facilitates can include transactions between the same individual at different points in time. Putting this in different words, let us say that a person is willing to pay for a good if she is willing to give up what would induce others (or, as in the case of our wine economy, herself at another point in time) to supply it. Then our claim is that the market gives each person, rational or irrational, what she wants and is willing to pay for, when she wants it and is willing to pay for it. 
Table 1: Price, consumption and utility in the three regimes

\begin{tabular}{|c|c|c|c|}
\hline & $\begin{array}{l}\text { autarky } \\
\text { regime }\end{array}$ & $\begin{array}{l}\text { precommitment } \\
\text { regime }\end{array}$ & $\begin{array}{l}\text { market } \\
\text { regime }\end{array}$ \\
\hline price $\left(p^{\mathrm{P}}\right.$ or $\left.p^{\mathrm{M}}\right)$ & - & $1 / 3$ & 1 \\
\hline \multicolumn{4}{|c|}{ actual consumption: } \\
\hline$\Lambda_{i, 1}^{1}$ & $1 / 2$ & $1 / 2$ & $3 / 4$ \\
\hline$\Lambda_{i, 2}^{1}$ & $1 / 2$ & $1 / 2$ & $1 / 4$ \\
\hline$\Lambda_{i, 1}^{2}$ & $1 / 2$ & $1 / 2$ & $1 / 4$ \\
\hline$\Lambda_{i, 2}^{2}$ & $1 / 2$ & $1 / 2$ & $3 / 4$ \\
\hline \multicolumn{4}{|c|}{$\begin{array}{l}\text { consumption planned } \\
\text { in period } 1 \text { : }\end{array}$} \\
\hline$\Lambda_{i, 1}^{2}$ & $1 / 2$ & $1 / 2$ & $3 / 4$ \\
\hline$\Lambda_{i, 2}^{2}$ & $1 / 2$ & $1 / 2$ & $1 / 4$ \\
\hline \multicolumn{4}{|l|}{ lifetime utility: } \\
\hline$u^{1}$ & -0.69 & -0.69 & -0.84 \\
\hline$u^{2}$ & -0.69 & -0.69 & -0.56 \\
\hline
\end{tabular}




\section{Appendix 1: Proof of the Market Opportunity Result}

Consider any storage economy and any market-clearing single-price regime $R$. Let $p \equiv\left(p_{1}\right.$, $\ldots, p_{g}$ ) be the vector of market prices in this regime. To initiate a proof by contradiction, suppose (Supposition 1 ) that $R$ does not satisfy the opportunity criterion. By the definition of 'market-clearing', $\chi(R)$ is feasible. Thus, there is some regime $\underline{R}$ with $O(\underline{R}) \supset O(R)$, and some feasible outcome $\underline{o} \in O(\underline{R}) \backslash O(R)$ such that $\underline{o}$ is non-dominated in $O(\underline{R})$. Let $q_{i}^{t}$ be $i$ 's $t$ period inheritance in $\underline{o}$, let $\underline{b}_{i}^{t}$ be $i$ 's $t$-period behaviour in $\underline{o}$, and so on. Because $\underline{o}$ is nondominated in $O(\underline{R})$, and because $O(\underline{R}) \supset O(R)$, each $\underline{b}_{i}^{t}$ must have the property that $i$ 's money consumption $\underline{\Lambda}_{i, 1}^{t}$ is greater than or equal to the money consumption he would have achieved in $R$, had his $t$-period inheritance been $q_{i}^{t}=q_{i}^{t}$ and had his $t$-period behaviour satisfied (i) $\Delta_{i, g}^{t}$ $=\underline{\Delta}_{i, g}^{t}$ for all $g>1$ and (ii) $\Delta_{i, 1}^{t}-\Lambda_{i, 1}^{t}=\underline{\Delta}_{i, 1}^{t}-\underline{\Lambda}_{i, 1}^{t}$. That is:

$$
\text { for all } i, t: \quad \underline{\Lambda}_{i, 1}^{t} \geq-\sum_{g=2}^{G} p_{g} \underline{\Delta}_{i, g}^{t}-\left(\underline{\Delta}_{i, 1}^{t}-\underline{\Lambda}_{i, 1}^{t}\right)
$$

Rearranging and using $p_{1}=1$ :

$$
\text { for all } i, t: \quad \sum_{g=1}^{G} p_{g} \underline{\Delta}_{i, g}^{t} \geq 0 \text {. }
$$

Suppose (Supposition 2) that (A2) is a strict inequality for some $i$ and $t$. Then:

$$
\sum_{i} \sum_{t} \sum_{g=1}^{G} p_{g} \underline{\Delta}_{i, g}^{t}>0
$$

But the feasibility of $\underline{o}$ implies

$$
\text { for all } g=1, \ldots, G: \quad \sum_{i} \sum_{t} \underline{\Delta}_{i, g}^{t}=0
$$

Since (A3) and (A4) are mutually contradictory, Supposition 2 is false. Thus

$$
\text { for all } i, t: \quad \sum_{g=1}^{G} p_{g} \underline{\Delta}_{i, g}^{t}=0 \text {. }
$$

But this condition is identical to (7), which defines opportunity functions in $R$ (see Section 3 above). Thus, $\underline{O} \in O(R)$, contrary to Supposition 1 . 


\section{Appendix 2: Alternative formulations of the opportunity criterion}

In this Appendix, we examine the relationship between the opportunity criterion as formulated in the current paper and the formulation used by Sugden (2004). Since Sugden's criterion is specified only for a one-period economy, we compare the two criteria as applied to that case. For ease of comparison, we use Sugden's notation and definitions.

The one-period economy consists of individuals $i=1, \ldots, n$ and goods $j=1, \ldots, m$, where $n>1$ and $m>1$. Each good is in fixed supply. A bundle is any non-negative $m$-tuple of quantities of goods. Any $n$-tuple of bundles $\mathbf{x} \equiv\left(\mathbf{x}_{1}, \ldots, \mathbf{x}_{n}\right)$ is an allocation; each $\mathbf{x}_{i}$ is the bundle held by individual $i$. The set of feasible allocations (that is, allocations that are consistent with the fixed supply conditions) is $X$. For each individual $i$ there is an opportunity set $O_{i}$, whose elements are bundles; the profile $\left(O_{1}, \ldots, O_{n}\right)$ is denoted $\mathbf{O}$. The Cartesian product of the $n$ opportunity sets is denoted $\times_{i} O_{i}$; its elements are allowable allocations. Allowable allocations are not necessarily feasible. However, suppose that each individual $i$ chooses $\mathbf{x}_{i}^{*} \in O_{i}$ such that $\mathbf{x}^{*}$ is feasible. Sugden's formulation of the opportunity criterion (which we will call OC1) is as follows:

OC1 is satisfied by $\left(\mathbf{O}, \mathbf{x}^{*}\right)$ if and only if, for every allocation $\mathbf{x}^{\prime} \neq \mathbf{x}^{*}$, either $\mathbf{x}^{\prime} \notin X$ or there is some consumer $j$ such that $\mathbf{x}_{j}^{\prime} \neq \mathbf{x}_{j}{ }^{*}$ and $\mathbf{x}_{j}^{\prime} \in O_{j}$.

In other words: every feasible allocation other than the one that has in fact come about assigns to some individual a bundle that that individual had, but did not take, the opportunity to acquire.

Sugden proves (as his 'Result 1') that every competitive equilibrium satisfies OC1. More precisely, let $\mathbf{z}$ be the allocation that represents individuals' endowments; this allocation exactly exhausts the supply of each good. A competitive equilibrium is a pair $(\mathbf{O}$, $\left.\mathbf{x}^{*}\right)$ which satisfies the following two conditions. First, there is a vector $\rho$ of non-negative prices, such that for each individual $i, O_{i}$ is the set of bundles whose value at those prices is no greater than the value of $\mathbf{z}_{i} ; \mathbf{x}_{i}$ is the bundle chosen by $i$ from this opportunity set. Second, for each good $j$, net excess demand is non-positive, and is zero if $\rho_{j}>0$. Notice that the first condition assumes free disposal, while the second condition assumes that disposal options are not taken up.

These two features of the definition of competitive equilibrium allow Sugden to avoid what we will call the recycling problem. For example, consider a two-consumer 
economy with a given $X$ and $\mathbf{z}$. Suppose that each individual has a strictly positive endowment of every good. Let $\mathbf{x}^{\prime}$ be a feasible allocation such that $\mathbf{x}_{1}{ }^{\prime}\left\langle\mathbf{z}_{1}\right.$ and $\mathbf{x}_{2}{ }^{\prime}>\mathbf{z}_{2}$. This allocation can be reached if individual 1 disposes of some of her endowments without taking anything in return, and if this surplus is 'recycled' to individual 2. A simple Walrasian economy, of the kind Sugden is modelling, does not provide a mechanism for such recycling. If $\mathrm{OC} 1$ is to be satisfied in such an economy, each individual must have options to dispose of her endowments but must reject those options.

As a preliminary to an alternative formulation of the opportunity criterion, we consider what is means for OC1 not to hold. Consider any $\left(\mathbf{O}, \mathbf{x}^{*}\right)$, where $\mathbf{x}^{*}$ is feasible, but such that $\mathrm{OC} 1$ is not satisfied. Then there is a feasible allocation $\mathbf{x}^{\prime} \neq \mathbf{x}^{*}$ such that for all $i$, either $\mathbf{x}_{i}^{\prime}=\mathbf{x}_{i}^{*}$ or $\mathbf{x}_{i}^{\prime} \notin O_{i}$. Because $\mathbf{x}^{\prime} \neq \mathbf{x}^{*}$, there must be some individual $k$ for whom $\mathbf{x}_{k}^{\prime} \notin$ $O_{k}$; thus, $\mathbf{x}^{\prime}$ is not allowable with respect to $\mathbf{O}$. Now suppose that, for each $i$, we define $O^{\prime}{ }_{i} \equiv$ $O_{i} \cup\left\{\mathbf{x}_{i}^{\prime}\right\}$. Clearly, $\mathbf{x}^{\prime}$ is allowable with respect to $\mathbf{O}^{\prime}$. Thus, in replacing $\mathbf{O}$ by $\mathbf{O}^{\prime}$, we have unambiguously expanded individuals' opportunity sets in such a way that some feasible allocation, previously not allowable, has become allowable. Or, to put this another way, when opportunity sets are as specified by $\mathbf{O}$, there is a putative transaction (namely moving to $\mathbf{x}^{\prime}$ ) that is feasible but which is not permitted, even with the consent of everyone who would be a party to that transaction. In this sense, $\mathbf{O}$ does not maximise individuals' opportunities.

This result suggests that it might be possible to formulate the opportunity criterion more transparently, as requiring the absence of putative transactions that are feasible but not permitted. However, because of the recycling problem, such a reformulation is not completely straightforward. Consider a two-consumer economy in which each individual has a strictly positive endowment of every good. Suppose that, as before, we were to assume both free disposal and that disposal options are not taken up. Let $\left(\mathbf{O}, \mathbf{x}^{*}\right)$ be a competitive equilibrium. Let $\mathbf{x}^{\prime}$ be a feasible allocation such that $\mathbf{x}_{1}{ }^{\prime}\left\langle\mathbf{z}_{1}\right.$ and $\mathbf{x}_{2}^{\prime}>\mathbf{z}_{2}$. Since $\mathbf{x}_{1}{ }^{\prime} \neq \mathbf{x}_{1}{ }^{*}$ and $\mathbf{x}_{1}{ }^{\prime} \in O_{1}$, there is no violation of OC1 $\left(\mathbf{x}_{1}{ }^{\prime}\right.$ has been rejected by individual 1$)$. Nevertheless, $\mathbf{x}^{\prime}$ is not allowable with respect to $\mathbf{O}\left(\mathbf{x}_{2}^{\prime}\right.$ is not in individual 2's opportunity set) but is allowable with respect to the relevant $\mathbf{O}^{\prime}$. The implication of this example is that if the spirit of $\mathrm{OC} 1$ is to be retained, it must be reformulated as the absence of putative transactions that are feasible and non-dominated but not permitted. 
For any individual $i$ and any opportunity set $O_{i}$, we define a bundle $\mathbf{x}_{i} \in O_{i}$ to be dominated with respect to $O_{i}$ if there is some bundle $\mathbf{x}_{i}{ }^{\prime} \in O_{i}$ such that $\mathbf{x}_{i}^{\prime}>\mathbf{x}_{i}$. An allocation $\mathbf{x}^{\prime}$ is dominated with respect to $\mathbf{O}$ if there is any $i$ for whom $\mathbf{x}_{i}{ }^{\prime}$ is dominated with respect to $O_{i}$. We can now formulate an alternative version of the opportunity criterion, OC2:

Consider any $\left(\mathbf{O}, \mathbf{x}^{*}\right)$ such that $\mathbf{x}^{*}$ is feasible. OC2 is satisfied if and only if, for every $\mathbf{O}^{\prime}$ for which $\times_{i} O_{i}{ }^{\prime} \supset \times_{i} O_{i}$, every feasible allocation $\mathbf{x}^{\prime} \in\left[\times_{i} O_{i}{ }^{\prime}\right] \backslash\left[\times_{i} O_{i}\right]$ is dominated with respect to $\mathbf{O}^{\prime}$.

In other words, it is not possible to expand the set of allowable allocations in such a way that some feasible allocation, not previously allowable, becomes both allowable and nondominated.

Notice that, given that $\mathbf{x}^{*}$ is feasible, OC2 makes no further reference to individuals' actual choices. Thus, to a greater extent than $\mathrm{OC} 1, \mathrm{OC} 2$ focuses on opportunity sets rather than on what is chosen from those sets. In order to avoid the recycling problem, and hence to be able to show that competitive equilibrium satisfies OC2, it is not necessary to assume free disposal. Nor is it necessary to require that $\mathbf{x}^{*}$ is non-dominated with respect to $\mathbf{O}$. For these reasons, we prefer $\mathrm{OC} 2$ to $\mathrm{OC} 1$ as a criterion of maximal opportunity. The criterion formulated in the current paper generalises OC2 so that it applies to multi-period storage economies, the one-period exchange economy being a special case.

\section{References}

Bernheim, Douglas and Antonio Rangel (2007). Toward choice-theoretic foundations for behavioral welfare economics. American Economic Review: Papers and Proceedings 97: 464-470.

Camerer, Colin, Samuel Issacharoff, George Loewenstein, Ted O'Donaghue and Matthew Rabin (2003). Regulation for conservatives: behavioral economics and the case for 'asymmetric paternalism'. University of Pennsylvania Law Review 151: 1211-1254. 
Hammond, Peter (2003) Equal rights to trade and mediate. Social Choice and Welfare 21: $181-193$.

Sugden, Robert (2004). The opportunity criterion: consumer sovereignty without the assumption of coherent preferences. American Economic Review, 94: 1014-1033.

Sugden, Robert (2007). The value of opportunities over time when preferences are unstable. Social Choice and Welfare 29: 665-682.

Sugden, Robert (2010). Opportunity as mutual advantage. Economics and Philosophy. 26: 47-68.

Sunstein, Cass R. and Richard H. Thaler (2003). Libertarian paternalism is not an oxymoron. University of Chicago Law Review, 70: 1159-1202.

Thaler, Richard H. and Cass R. Sunstein (2008). Nudge: Improving Decisions about Health, Wealth, and Happiness. Yale University Press. 